\title{
The application of the artificial neural network in analysing the spread quality
}

\author{
Marko Petkovic ${ }^{1}$, Marija Blagojevic $^{2}$, and Vladimir Mladenovic ${ }^{2 *}$ \\ ${ }^{1}$ Faculty of Agronomy, University of Kragujevac, Food Technology Department, 32000 Čačak, \\ Serbia \\ ${ }^{2}$ Faculty of Technical Science in Čačak, University of Kragujevac, Information Technology \\ Department, 32000 Čačak, Serbia
}

\begin{abstract}
In this paper, we introduce a new approach in food processing using an artificial intelligence. The main focus is simulation of production of spreads and chocolate as representative confectionery products. This approach aids to speed up, model, optimize, and predict the parameters of food processing trying to increase quality of final products. An artificial intelligence is used in field of neural networks and methods of decisions.
\end{abstract}

\section{Introduction}

Nowadays, with World's population growth, an ever-growing need for resources such as food, the food industry has the challenge to speed up the production process, increases the quantity, and raises the quality of the nutritious products. For these reasons, each food processing company must increase its efficiency. Food processing is the transformation of agricultural products into food, or of one form of food into other forms of food. It represents one of the major futures of food manufacturing sectors. Food processing includes many forms of processing foods, from grinding grain to make raw flour to home cooking to complex industrial methods used to make convenience foods [1]. Agricultural and food products are connected in terms of raw agricultural product (such as grains), obtained from different grain varieties and agro-technical parameters, through the grounded flour and final product (bread, biscuit, cake). The wide range of food processing parameters discussed for the model formulation, make them useful and practical for designing the food processing. The effects of different food processing parameters, such as temperature, ingredient combination or ratio, processing time, type of packaging etc. can be tested [2]. The food expectations of grain products are higher nowadays: high nutritional values, beneficial health effects, longevity, safety, practical packaging, not seasonal food, easy to prepare and affordable. Fruits and vegetables, as a row agricultural material, are food with potentially high levels of antioxidants (such as flavonoids). High levels of antioxidants are present in a fresh fruit. Food processing keeps the high levels of antioxidants in fruits and vegetables drying them different process parameters (temperature, dehydration type, various conditions of fruit/vegetable cultivation etc.).

\footnotetext{
* Corresponding author: vladimir.mladenovic@ftn.kg.ac.rs
} 
The artificial-intelligence-based methods (AIBM) encompass a group of algorithms based on artificial neural networks, and machine learning, which can efficiently and quickly enable the development of methods that provide predicting food parameters. The effects of AIBMs can be seen in various scientific fields. The AIBMs are successful in the analysis of food quality based on the color, shape, texture, functionality etc. and predict the optimal model from seed to feed. The AIBMs explain the model of predicting process output with a very high coefficient of determination. The AIBMs develop to predict the best sensory, physical, chemical, and thermal characteristics of spreads or chocolate for a wide range of experimental conditions (temperature, grinding speed, grinding time, ingredient combination or ratio, characteristics of ball mill refiner). This model is able to predict the experimental kinetics of spread processing, its implementation for design and optimization of food processing.

The AIBMs explore the data using different algorithms of artificial intelligence to find the one that best predicts the experimental kinetic of the drying process and total antioxidant capacity of food. It is allowing the producers to analyze consumers' choices to find out which flavor or taste, or some other parameters, they want and predict what combinations could be most appropriate. In this project, the AIMBs will be strongly used for development of algorithms and predict for food processing to speed up food production, and increase the parameters for quality of food.

\section{Sugar free food and sugar alcohols}

One of the primary satisfactions of consumers is that spreads must be sweet taste without body weight effects increasing. In that sense, the replacement of nutritive sweeteners with other low-energy sweeteners (such as polyols) can change the sensory characteristics of the basic product. Proper selection of raw materials, as well as proper management of the technological process, can be obtained the products with optimal sensory properties [2].

Low-energy foods, or products with reduced energy value, are very popular among the consumers. There is a need for developing new and enriched existing products nowadays. The consumers' awareness is raised. They want to primarily satisfy the need for the sweet taste, as well as for maintaining or reducing their body weight, without any consequences for your health [3].

In addition to traditional carbohydrate sweeteners sucrose (saccharose), glucose, fructose, maltose, lactose, honey etc, the spreads can be produced by noncarbohydrate sweeteners such as sugar alcohols (polyols). The polyols include sorbitol (E 420), mannitol (E 421), isomalt (E 953), maltitol (E 965), lactitol (E 966), xylitol (E 967) and erythritol (E 968). Polyols are extremely stable at high temperatures and do not react with amino acids. For the most part, polyols have around $40 \%$ less energy $(10 \mathrm{~kJ} / \mathrm{g})$, do not participate in caramelization and Maillard reactions, are not fermentable and have a pleasant and neutral taste. The disadvantage of polyols is the laxative effect $[4,5]$.

The sweetness of sugar alcohols (polyols), is shown in Figure 1 [6]. 


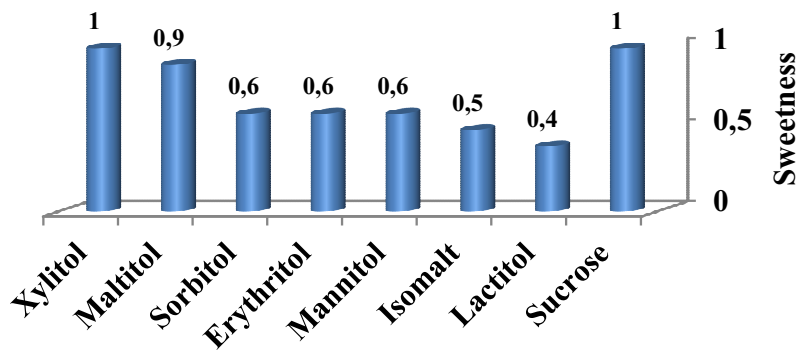

Fig. 1. The sweetness of polyols

When we look at the physical and chemical properties of sucrose, and we consider chocolate or spreads, the most ideal decision of sucrose substitute is maltitol. Maltitol (E $965)$ is a white crystalline powder, which causes a mellow cooling impact, with physicochemical attributes like sucrose. As a sucrose substitute, the innovative parameters of chocolate and spread production shouldn't be changed. The daily intake of maltitol relies upon purchaser living being condition, its age, sex, and so on, and is $50 \mathrm{~g}[6,7]$.

Replacement of nutritive sweeteners with other low-energy sweeteners (such as polyols) can change the sensory characteristics of the basic product. Proper selection of raw materials, as well as proper management of the technological process, can be obtained the products with optimal sensory properties [2].

\subsection{Technology of spread and chocolate}

Spreads, such as chocolate, do not contain cocoa butter (which requires a conching and tempering stage), however special vegetable fats (sunflower, soy, rapeseed etc.), so spread production is less expensive and less requesting. In laboratory and industry, ball mill refiner is most often used. The process parameters are the temperature, the mixer speed rotation, the diameter/number of balls, the speed of spread recirculation, the fat/solid content, the water content, the type/amount of emulsifiers and the particle size distribution. The mixture of melted fat and powder ingredients circulate through the ball mill refiner, until the optimum particle size is obtained (particle size reduction, the largest particles $<30 \mu \mathrm{m}$, optimum $20-22 \mu \mathrm{m})$ [2].

\section{New methods of artificial intelligence in food processing}

The artificial-intelligence-based methods (AIBM) encompass a group of algorithms based on artificial neural networks and machine learning. Altogether there are many fields of artificial intelligence. Since its inception, artificial intelligence is still a field that is difficult to define precisely. But the notion of artificial intelligence is most often associated with a Turing-based approach, a cognitive modelling approach, a rational-based approach using formal logic, and an approach based on rational agents. The effects of AIBMs can be seen in various scientific fields such as industrial process management, monitoring of the operation of medical devices, autonomous movement of vehicles (on land and water), automatic pilots, satellite control, monitoring installations, operational and tactical management of military operations in the battlefield, analysis of complex financial transactions, oil platforms, for the detection of oil wells. 
The AIBM uses artificial neural networks for processing food. Feedback must exist in order to the neural network learns to recognize and classify terms. The first step is to train the data network. This process involves comparing the output generated by the network with the output that should be generated. The difference between these outputs is used to correct the network weight in the network. First, the weights are corrected closer to the output layer, moving backward, all the way to the input layer. This process is named back propagation. The back propagation, for some time, allows the network to learn, reducing the difference between actual and desired results, until these two values fit. When a network is being trained with a sufficient amount of data, a new dataset has introduced that lack the attribute by which the classification is performed in order to test its efficiency [8]. The network will attempt to categorize new cases depending on how it is trained.

In this paper, the following steps were used to create a neural network and data processing. The procedure includes the following steps:

- data selection,

- data pre-processing,

- modelling of neural network and

- testing the network through the dataset processing.

In the next section, a concrete analysis, results, and discussion are presented.

\section{Analysis, results and discussion}

\subsection{Analysis and results}

The analysis is done by collecting the dataset for the spread making process. The following parameters are taken as the input variables: grinding time in minutes $(t)$, temperature in ${ }^{\circ} \mathrm{C}$ $(T)$, rotational speed of the mixer rpm - rotate per minute $(V)$, maltitol content in $\%\left(C_{\mathrm{M}}\right)$, sucrose content in $\%\left(C_{\mathrm{s}}\right)$, sum of maltitol and sucrose in $\%\left(C_{\mathrm{Ms}}\right)$, external appearance $(e)$, texture $\left(t_{\mathrm{x}}\right)$, chewyness $(c)$, taste $\left(t_{\mathrm{a}}\right)$, and flavor $(f)$. All values are obtained based on premade spreads to obtain quality indicators, the correction of parameters is done at external appearance $(e)$, texture $\left(t_{\mathrm{x}}\right)$, chewyness $(c)$, taste $\left(t_{\mathrm{a}}\right)$, and fragrance $(f)$, and this is so [11$14]$.

$$
\begin{aligned}
& E=0.6 \cdot e \\
& T_{\mathrm{x}}=0.8 \cdot t_{\mathrm{x}} \\
& C=1 \cdot \mathrm{c} \\
& T_{\mathrm{a}}=0.6 \cdot t_{\mathrm{a}} \\
& F=1 \cdot f
\end{aligned}
$$

The quality indicator is obtained by summarize corrected parameters as $[\mathbf{1 5}, \mathbf{1 6}]$

$$
\Sigma=E+T_{\mathrm{x}}+C+T+F
$$

The main task of research is to find the most optimal solution that provides the best recipe for the quality indicator in the range between 17.6 and 20 [17-22].

All parameter values are shown in the table 1: 
Table 1. Measured dataset

\begin{tabular}{|c|c|c|c|c|c|c|c|c|c|c|c|c|c|c|c|c|c|}
\hline $\begin{array}{c}\text { ID } \\
\text { scream }\end{array}$ & $\mathrm{t}$ & $\mathrm{T}$ & V & $\mathrm{C}_{\mathrm{M}}$ & $\mathrm{C}_{\mathrm{s}}$ & $\mathrm{C}_{\mathrm{Ms}}$ & e & $t_{x}$ & $\mathrm{c}$ & $\mathrm{t}_{\mathrm{a}}$ & f & E & $\mathrm{T}_{\mathrm{x}}$ & $\mathrm{C}$ & $\mathrm{T}_{\mathrm{a}}$ & F & $\Sigma$ \\
\hline S3060 & 150 & 30 & 60 & 0 & 47.4 & 47.4 & 4 & 4 & 4 & 5 & 4 & 2.4 & 3.2 & 4 & 3 & 4 & 16.6 \\
\hline $\begin{array}{l}\text { S3080 } \\
\end{array}$ & 150 & 35 & 80 & 0 & 47.4 & $\begin{array}{l}47.4 \\
\end{array}$ & 3 & 3.5 & 4 & 4.5 & 4 & 1.8 & 2.8 & 4 & 2.7 & 4 & 15.3 \\
\hline S30100 & 150 & 40 & 100 & 0 & 47.4 & 47.4 & 2.5 & 3 & 4 & 5 & 4.5 & 1.5 & 2.4 & 4 & 3 & 4.5 & 15.4 \\
\hline S3560 & 150 & 30 & 60 & 0 & 47.4 & 47.4 & 3.5 & 3.5 & 4 & 4.5 & 4.5 & 1.5 & 2.8 & 4 & 2.7 & 4.5 & 15.5 \\
\hline S3580 & 150 & 35 & 80 & 0 & 47.4 & 47.4 & 3.5 & 3.5 & 4 & 5 & 4.5 & 1.5 & 2.8 & 4 & 3 & 4.5 & 15.8 \\
\hline S35100 & 150 & 40 & 100 & 0 & 47.4 & 47.4 & 3.5 & 3 & 4 & 4 & 4.5 & 1.5 & 2.4 & 4 & 2.4 & 4.5 & 14.8 \\
\hline S4060 & 150 & 30 & 60 & 0 & 47.4 & 47.4 & 3.5 & 3.5 & 4.5 & 5 & 4.5 & 1.5 & 2.8 & 4.5 & 3 & 4.5 & 16.3 \\
\hline S4080 & 150 & 35 & 80 & 0 & 47.4 & 47.4 & 3.5 & 3.5 & 4.5 & 5 & 4.5 & 1.5 & 2.8 & 4.5 & 3 & 4.5 & 16.3 \\
\hline S40100 & 150 & 40 & 100 & 0 & 47.4 & 47.4 & 3.5 & 4.5 & 4.5 & 5 & 5 & 2.1 & 3.6 & 4.5 & 3 & 5 & 18.2 \\
\hline M3060 & 150 & 30 & 60 & 47.4 & 0 & 47.4 & 3.5 & 4 & 4 & 4 & 4 & 2.1 & 3.2 & 4 & 2.4 & 4 & 15.7 \\
\hline M3080 & 150 & 35 & 80 & 47.4 & 0 & 47.4 & 4.5 & 4.5 & 4 & 4.5 & 4 & 2.7 & 3.6 & 4 & 2.7 & 4 & 17.0 \\
\hline M30100 & 150 & 40 & 100 & \begin{tabular}{|l|l|}
47.4 \\
\end{tabular} & $\overline{0}$ & \begin{tabular}{|l|l|}
47.4 \\
\end{tabular} & 4.5 & 5 & 4 & 4 & 4.5 & 2.7 & 4 & 4 & 2.4 & 4.5 & 17.6 \\
\hline M3560 & 150 & 30 & 60 & 47.4 & 0 & 47.4 & 4 & 3.5 & 3.5 & 4 & 4 & 2.4 & 2.8 & 3.5 & 2.4 & 4 & 15.1 \\
\hline M3580 & 150 & 35 & 80 & 47.4 & 0 & 47.4 & 4.5 & 4.5 & 4 & 5 & 5 & 2.7 & 3.6 & 4 & 3 & 5 & 18.3 \\
\hline M35100 & 150 & 40 & 100 & 47.4 & 0 & 47.4 & 5 & 4.5 & 4.5 & 5 & 5 & 3 & 3.6 & 4.5 & 3 & 5 & 19.1 \\
\hline M4060 & 150 & 30 & 60 & 47.4 & 0 & 47.4 & 3.5 & 4 & 4 & 4.5 & 5 & 2.1 & 3.2 & 4 & 2.7 & 5 & 17.0 \\
\hline M4080 & 150 & 35 & 80 & 47.4 & 0 & 47.4 & 4 & 4.5 & 4.5 & 4.5 & 5 & 2.4 & 3.6 & 4.5 & 2.7 & 5 & 18.2 \\
\hline M40100 & 150 & 40 & 100 & 47.4 & 0 & 47.4 & 4 & 5 & 4.5 & 4.5 & 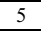 & 2.4 & 4 & 4.5 & 2.7 & 5 & 18.6 \\
\hline $\begin{array}{l}\text { SM3060 } \\
\end{array}$ & 150 & 30 & 60 & 14.22 & 33.18 & 47.4 & 3.5 & 3.5 & 4 & 4 & 4 & 2.1 & 2.8 & 4 & 2.4 & 4 & 15.3 \\
\hline SM3080 & 150 & 35 & 80 & 14.22 & 33.18 & 47.4 & 3.5 & 3.5 & 4 & 4 & 4.5 & 2.1 & 2.8 & 4 & 2.4 & 4.5 & 15.8 \\
\hline SM30100 & 150 & 40 & 100 & 14.22 & 33.18 & 47.4 & 3.5 & 3.5 & 4 & 4.5 & 4.5 & 2.1 & 2.8 & 4 & 2.7 & 4.5 & 16.1 \\
\hline SM3560 & 150 & 30 & 60 & 14.22 & 33.18 & 47.4 & 3.5 & 3.5 & 4 & 4 & 4 & 2.1 & 2.8 & 4 & 2.4 & 4 & 15.3 \\
\hline SM3580 & 150 & 35 & 80 & 14.22 & 33.18 & 47.4 & 3.5 & 3.5 & 4 & 4 & 4 & 2.1 & 2.8 & 4 & 2.4 & 4 & 15.3 \\
\hline SM35100 & 150 & 40 & 100 & 14.22 & 33.18 & 47.4 & 3.5 & 3.5 & 4.5 & 4 & 4.5 & 2.1 & 2.8 & 4.5 & 2.4 & 4.5 & 16.3 \\
\hline SM4060 & 150 & 30 & 60 & 14.22 & 33.18 & 47.4 & 4 & 3.5 & 4.5 & 4 & 4 & 2.4 & 2.8 & 4.5 & 2.4 & 4 & 16.1 \\
\hline SM4080 & 150 & 35 & 80 & 14.22 & 33.18 & 47.4 & 4 & 4.5 & 4.5 & 4.5 & 5 & 2.4 & 3.6 & 4.5 & 2.7 & 5 & 18.2 \\
\hline SM40100 & 150 & 40 & 100 & 14.22 & 33.18 & 47.4 & 4 & 3.5 & 4 & 4 & 4.5 & 2.4 & 2.8 & 4 & 2.4 & 4.5 & 16.1 \\
\hline MS3060 & 150 & 30 & 60 & 33.18 & 14.22 & 47.4 & 4.5 & 4.5 & 4.5 & 4 & 4 & 2.7 & 3.6 & 4.5 & 2.4 & 4 & 17.2 \\
\hline MS3080 & 150 & 35 & 80 & 33.18 & 14.22 & 47.4 & 4 & 4.5 & 4.5 & 4 & 4.5 & 2.7 & 3.6 & 4.5 & 2.4 & 4.5 & 17.7 \\
\hline MS30100 & 150 & 40 & 100 & 33.18 & 14.22 & 47.4 & 4 & 4 & 4 & 4 & 4.5 & 2.7 & 3.2 & 4 & 2.4 & 4.5 & 16.8 \\
\hline $\begin{array}{l}\text { MS3560 } \\
\end{array}$ & 150 & 30 & 60 & 33.18 & 14.22 & 47.4 & 4 & 4.5 & 4.5 & 4 & 4 & 2.7 & 3.6 & 4.5 & 2.4 & 4 & 17.2 \\
\hline MS3580 & 150 & 35 & 80 & 33.18 & 14.22 & 47.4 & 4 & 4.5 & 5 & 4 & 4.5 & 2.7 & 3.6 & 5 & 2.4 & 4.5 & 18.2 \\
\hline MS35100 & 150 & 40 & 100 & 33.18 & 14.22 & 47.4 & 4 & 5 & 5 & 4 & 4.5 & 2.7 & 4 & 5 & 2.4 & 4.5 & 18.6 \\
\hline MS4060 & 150 & 30 & 60 & 33.18 & 14.22 & 47.4 & 4 & 4.5 & 4 & 4 & 4.5 & 2.7 & 3.6 & 4 & 2.4 & 4.5 & 17.2 \\
\hline MS4080 & 150 & 35 & 80 & 33.18 & 14.22 & 47.4 & 4 & 5 & 5 & 4 & 4.5 & 2.7 & 4 & 5 & 2.4 & 4.5 & 18.6 \\
\hline MS40100 & 150 & 40 & 100 & 33.18 & 14.22 & 47.4 & 4 & 4 & 4 & 4 & 4 & 2.4 & 3.2 & 4 & 2.4 & 4 & 16.0 \\
\hline
\end{tabular}

The laboratory ball mill is a vertical cylinder, with double walls through which water circulates. In the middle of the mill is a shaft with a mixer and blades, which moves the stainless steel balls. The characteristics of laboratory ball mill was: capacity $5 \mathrm{~kg}$, stainless steel balls $(9.1 \mathrm{~mm}$ diameter, $30 \mathrm{~kg}$ weight), the speed of spread recirculation is $10 \mathrm{~kg} / \mathrm{h}$, the refining (grinding) time is 150 minutes), the temperature ratio $\left(30^{\circ} \mathrm{C}, 35^{\circ} \mathrm{C}, 40^{\circ} \mathrm{C}\right)$ the mixer speed rotation (clockwise $-60,80,100 \mathrm{r} / \mathrm{min}$ ). The basic ingredients for spreads are: sweetener $47.4 \%$, palm fat $36 \%$ cocoa powder $7 \%$, whole milk powder $7 \%$, soy flour $2 \%$, lecithin $0.5 \%$, flavor $0.1 \%$. The sweetener was maltitol $(100 \%)$, sucrose $(100 \%)$ and a combination of maltitol/sucrose $(70 / 30 \%, 30 / 70 \%$ ratio). Spreads were produced at different temperatures $\left(30^{\circ} \mathrm{C}, 35^{\circ} \mathrm{C}, 40^{\circ} \mathrm{C}\right)$ and mixer speed rotation (clockwise $-60,80$, $100 \mathrm{r} / \mathrm{min}$ ). Spread with $100 \%$ maltitol, produced at temperature $30^{\circ} \mathrm{C}$ and $60 \mathrm{r} / \mathrm{min}$ is labelled as "M-30-60" [23]. Spreads, marked as S-40-100, M-30-100, M-35-80, M-35$100, \mathrm{M}-40-80, \mathrm{M}-40-100$, MS-30-80, MS-35-80, MS-35-100, MS-40-80 and SM-4080 , have excellent quality (points 17.6-20.0).

We utilize a standard multi-layer perceptron (MLP) with one output node and tangent sigmoidal transfer function [24]. Tests are performed by generating different types of neural network and comparative results. It ensures that the depth of the network of 20 layers gives the best results. The obtained results for the neural network test overcomes of the size of this paper so cannot be presented.

A multilayer perceptron, which is one of the back-propagation neural networks, has been applied. The back propagation training algorithm has been used. The neural network 
algorithm is used to create a network that, in this study, can contain one layers of neurons: an input layer, a hidden layer (which is optional), and an output layer.

- Input layer (34.02.07 in ISO/IEC 2382-34:1999 [2525]) defines all the input attribute values for the data mining model. Input parameters are: chew, texture, grinding time, content of maltitol, code of spreading, speed of rotation of the mixer, temperature, content of sucrose, external appearance, taste and smell.

-Hidden layer (34.02.10 in ISO/IEC 2382-34:1999, [2525]) contains neurons which receive input from the input layer and forward them to the output layer. In this layer, weights are assigned to input neurons.

-Output layer (34.02.08 in ISO/IEC 2382-34:1999, [25]) contains neurons which represent the attribute values that we are predicting. In the approach used here, spreading quality.

For the model evaluation, authors have used $30 \%$ of the dataset for testing and $70 \%$ of the dataset for training. The model was trained with the data obtained from measured parameters from 36 different types of spreading. Model was tested with new data that had not been used during training. The decision tree is obtained those points to the optimal recipe values for a predefined range dataset of excellent quality. This is depicted in figure 2 .

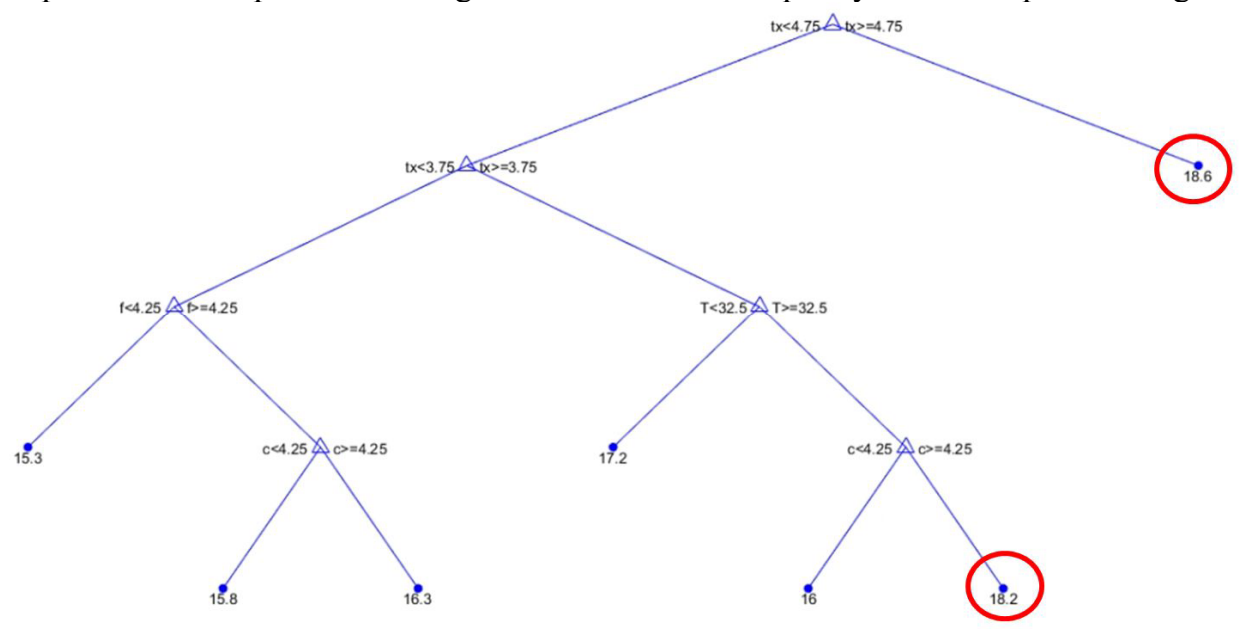

Fig. 2. Decision tree

\subsection{Discussion}

Spreads, produced on produced on temperature $35^{\circ} \mathrm{C}$ and $40^{\circ} \mathrm{C}$ and mixer speed rotation 80 $\mathrm{r} / \mathrm{min}$ and $100 \mathrm{r} / \mathrm{min}$, have excellent sensory properties. Increasing the process parameters, the excellent sensory properties of spreads are archived. The temperature and mixer speed rotation are optimized as the best suggestion for process parameters using by AIBM. The decision tree provides two values of quantity indicator: 18.2 and 18.6. Comparing AIBM optimization parameters and point of sensory characteristics, the spreads have points 18.2 and 18.6, such as: S-40-100, M-40-80, M-40-100, SM-40-80, MS-35-80, MS-35-100 and MS-40-80. This means that the AIBM network gives the following guidelines:

1. if we want to produce spreads only with sucrose, we should choose the highest values of process parameters

2. if we want to produce spreads only with maltitol, we should choose the highest temperature and the middle and highest mixer speed rotation parameters 
3. if we want to produce spreads with $70 \%$ sucrose and $30 \%$ of maltitol, we should choose the highest temperature and the middle mixer speed rotation parameter

4. if we want to produce spreads with $30 \%$ sucrose and $70 \%$ of maltitol, we should choose the middle and the highest process parameters.

This study was supported by the Serbian Ministry of Education and Science, TR 32023.

\section{References}

1. A. Grumezescu, A. M. Holban, Food Processing for Increased Quality and Consumption, Academic Press (2018)

2. M. Petkovic, B. Pajin, J Tomic, A. Torbica, Z. Serse, D. Zaric, Chemical Industry, 66, 385 (2012) DOI: 10.2298/HEMIND1 10902094P]

3. L. Bobroff, Ed.: K.W. O'Neil and R.L. Peterson, F.L. Sarasota, Book chapter: Nutrition and Diet, Handbook: Optimal Aging LLC, 627 (2004)

4. N. O'Brien, $3^{\text {th }}$ ed. Alternative Sweeteners (World Wide Web, New York, 2001)

5. F. Douglas, Formulation and production of chewing and bubble gum (Kennedy's Publications Ltd, London, 2006)

6. A. L. Nelson, Sweeteners: Alternative (Eagan Press, Minnesota, 2000)

7. M. E. Embuscado, S. K. Patil, Reduced-calorie sweeteners, (World Wide Web, New York, 2001)

8. M. Awad, R. Khanna, Deep Neural Networks. In: Efficient Learning Machines. (Apress, Berkeley, CA, 2015)

9. B. Pajin, A. Fistes, LJ. Dokic, I. Nikolic, Chem. Ind. J., 67 (5), 747 (2013)

10. B. Pajin, A. Fistes, J. Petrovic, A. Torbica, Optimization of the ball mill processing parameters in the fat filling Production CICEQ, 23 (2), 197 (2017)

11. SRPS ISO 5496:2014 - Sensory analysis -- Methodology -- Initiation and training of assessors in the detection and recognition of odours

12. SRPS EN ISO 10399:2018 - Sensory analysis - Methodology - Duo-trio test (ISO 10399:2017)

13. SRPS ISO 8588:2018 - Sensory analysis -- Methodology -- "A" - "not A" test

14. SRPS ISO 8587:2013/A1:2016 - This International Standard describes a method for sensory evaluation with the aim of placing a series of test samples in rank order

15. SRPS EN ISO 4120:2012 - Sensory analysis - Methodology - Triangle test (ISO 4120:2004)

16. SRPS EN ISO 5495:2012/ A1:2016 - Sensory analysis - Methodology - Paired comparison test (ISO 5495:2005/Amd 1:2016)

17. SRPS ISO 5496:2014 - Sensory analysis -- Methodology -- Initiation and training of assessors in the detection and recognition of odours

18. SRPS EN ISO 10399:2018 - Sensory analysis - Methodology - Duo-trio test (ISO 10399:2017)

19. SRPS ISO 8588:2018 - Sensory analysis -- Methodology -- "A" - "not A" test

20. SRPS ISO 8587:2013/A1:2016 - This International Standard describes a method for sensory evaluation with the aim of placing a series of test samples in rank order 
21. SRPS EN ISO 4120:2012 - Sensory analysis - Methodology - Triangle test (ISO 4120:2004)

22. SRPS EN ISO 5495:2012/ A1:2016 - Sensory analysis - Methodology - Paired comparison test (ISO 5495:2005/Amd 1:2016)

23. PETKOVIC M., The effects of producing parameters on physical properties, thermal characteristics and quality of spreads with maltitol [thesis]. Novi Sad; Faculty of Technology University of Novi Sad; (2012)

24. HAYKIN S. O., Neural Networks and Learning Machines, $3^{\text {rd }}$ Edn. Upper Saddle River, NJ: Pearson/Prentice Hall, (2009)

25. ISO/IEC 2382-34:1999 Information technology - Vocabulary - Part 34: Artificial intelligence -Neural Networks (1999)

26. C. Draper, R. Reichle, R. Jeu, V. Naemi, R. Parinussa, W. WAGNER, Remote Sens. Environ., 137, 288 (2013)

27. Ke-Lin Du, M. N. S. Swamy, Neural Networks and Statistical Learning (SpringerVerlag, London, 2014)

28. J. Schmidhuber, Neural Networks, 61, 85 arXiv:1404.7828 Freely accessible. doi:10.1016/j.neunet.2014.09.003. PMID 25462637 (2015)

29. D. Pokrajac, K. Sudler, P. Edamatsu, T. Hardee, $13^{\text {th }}$ Symposium on Neural Networks and Applications (NEUREL), 1 (2016) DOI: 10.1109/NEUREL.2016.7800124 